\title{
Lipschitz structure and minimal metrics on topological groups
}

\author{
Christian Rosendal
}

\begin{abstract}
We discuss the problem of deciding when a metrisable topological group $G$ has a canonically defined local Lipschitz geometry. This naturally leads to the concept of minimal metrics on $G$, that we characterise intrinsically in terms of a linear growth condition on powers of group elements.

Combining this with work on the large scale geometry of topological groups, we also identify the class of metrisable groups admitting a canonical global Lipschitz geometry.

In turn, minimal metrics connect with Hilbert's fifth problem for completely metrisable groups and we show, assuming that the set of squares is sufficiently rich, that every element of some identity neighbourhood belongs to a 1-parameter subgroup.
\end{abstract}

\section{Introduction}

The present note deals with the problem of deciding which metrisable topological groups have a well-defined local geometry intrinsic to the topological group structure. To make this problem more precise, let us recall that a metrisable topological group is a topological group $G$ whose topology may be induced by some metric, which then is said to be compatible with the topology on $G$. Thus, the metric itself is not part of the given data. These groups where characterised in fundamental papers by G. Birkhoff [1] and S. Kakutani [7]; namely, a Hausdorff topological group $G$ is metrisable if and only if it is first countable. Moreover, such a group necessarily

The research was partially supported by a Simons Foundation Fellowship (Grant \#229959) and by the NSF (DMS 1201295 \& DMS 1464974). The author is grateful for very helpful conversations with I. Goldbring and for the detailed comments by the referees

Key words and phrases: metrisable groups, left-invariant metrics, Hilbert's fifth problem, Lipschitz structure.

2010 Mathematics Subject Classification: primary 22A10; secondary 03E15. 
admits a compatible left-invariant metric $d$, i.e., so that $d(h g, h f)=d(g, f)$ for all $g, f, h \in G$.

An easy calculation shows that, if $d$ and $\partial$ are compatible left-invariant metrics on a topological group $G$, then the identity map id: $(G, \partial) \rightarrow(G, d)$ is always uniformly continuous and hence, by symmetry, a uniform homeomorphism. This is of course also a reflection of the fact that both $d$ and $\partial$ will metrise the left-uniform structure on $G$. However, unless further assumptions are added, there is generally no control on the modulus of uniform continuity of the mapping. The problem is thus to decide which, if any, of the compatible left-invariant metrics on $G$ determine a canonical local geometric structure on the group. At least up to local bi-Lipschitz equivalence, this is solved if $G$ admits a minimal metric in the following sense.

Definition 1. A metric $d$ on a topological group $G$ is said to be minimal if it is compatible, left-invariant and, for every other compatible left-invariant metric $\partial$ on $G$, the map

$$
\text { id }:(G, \partial) \longrightarrow(G, d)
$$

is Lipschitz in a neighbourhood of the identity, i.e., if there is an identity neighbourhood $U$ and a constant $K$ so that

$$
d(g, f) \leqslant K \cdot \partial(g, f)
$$

for all $g, f \in U$.

Let us first observe that, if $U$ and $K$ are as above, then id: $(G, \partial) \rightarrow(G, d)$ is locally $K$-Lipschitz. For given $h \in G$ and $v, w \in U$, note that

$$
d(h v, h w)=d(v, w) \leqslant K \cdot \partial(v, w)=\partial(h v, h w),
$$

so the identity map is $K$-Lipschitz on the neighbourhood $h U$ of $h$. It follows immediately that any two minimal metrics on $G$ are locally bi-Lipschitz and thus identify a canonical local geometric or, more specifically, local Lipschitz structure on $G$.

The concept of an intrinsic Lipschitz structure on a topological object is of course common to other areas. For example, a well-known result due to D. Sullivan [14] states that, except for $n=4$, any topological $n$-manifold $M$ admits a Lipschitz structure, that is, an atlas $\left\{\phi_{i}: U_{i} \rightarrow \mathbb{R}^{n}\right\}$ whose transition maps are locally Lipschitz. Moreover, any two such Lipschitz structures are related by a locally bi-Lipschitz homeomorphism of $M$. The local Lipschitz structure identified by a minimal metric is even more rigid, since any two minimal metrics are locally biLipschitz via the identity map.

We remark that, unless we accept to force the metric $d$ to be bounded, the local minimality of Definition 1 really describes the strongest notion of minimality 
possible. Indeed, if $d$ is unbounded, then $\sqrt{d}$ is a compatible left-invariant metric, while id: $(G, \sqrt{d}) \rightarrow(G, d)$ is not Lipschitz for large distances.

Note also that, at least for short distances, there is no maximal metric unless $G$ is discrete. That is, if $G$ is non-discrete and $d$ is any compatible left-invariant metric, then the mapping id: $(G, d) \rightarrow(G, \sqrt{d})$ will not be Lipschitz for short distances.

Observation 2. (Independence of localising set) Another helpful observation is that, if $d$ is minimal and $U$ is a fixed $d$-bounded identity neighbourhood, then, for every compatible left-invariant $\partial$, the map

$$
\mathrm{id}:(U, \partial) \longrightarrow(U, d)
$$

is Lipschitz, i.e., the localising set $U$ can be chosen independently of $\partial$. To see this, suppose that $\partial$ is given and pick an identity neighbourhood $V$ and a constant $K$ so that id: $(V, \partial) \rightarrow(V, d)$ is $K$-Lipschitz. Pick $\alpha>0$ so that the open ball

$$
B_{\partial}(\alpha)=\{g \in G \mid \partial(g, 1)<\alpha\}
$$

is contained in $V$. Then, for any two elements $g, f \in U$, either $g^{-1} f \in V$, whence

$$
d(g, f)=d\left(1, g^{-1} f\right) \leqslant K \cdot \partial\left(1, g^{-1} f\right)=K \cdot \partial(g, f),
$$

or $g^{-1} f \notin V$, whence

$$
d(g, f) \leqslant \operatorname{diam}_{d}(U) \leqslant \frac{\operatorname{diam}_{d}(U)}{\alpha} \cdot \partial(g, f) .
$$

In other words, id: $(U, \partial) \rightarrow(U, d)$ is Lipschitz with constant

$$
K+\frac{\operatorname{diam}_{d}(U)}{\alpha} .
$$

From this observation, it follows immediately that any two bounded minimal metrics are bi-Lipschitz equivalent. Nevertheless, in many cases, a better global Lipschitz structure can be identified that captures the large scale geometry of the group. We return to this in Theorem 14.

One way to think about minimal metrics is via the decay of the balls $B_{d}(\alpha)$ as $\alpha \rightarrow 0$. Namely, minimality of $d$ simply expresses that, if $\partial$ is another compatible left-invariant metric on $G$, then, for some $\lambda=\lambda(\partial)>0$, we have

$$
B_{\partial}(\lambda \cdot \alpha) \subseteq B_{d}(\alpha)
$$

whenever $\alpha \leqslant 1$. So, up to the rescaling by $\lambda$, the $d$-balls $B_{d}(\alpha)$ are as large as possible as $\alpha$ decreases to 0 .

Whereas minimality of a metric is a relative notion, i.e., defined in terms of comparisons with other compatible left-invariant metrics on the group, the main result 
of our note furnishes an internal characterisation of minimality without reference to other metrics. Namely, we characterise the minimal metrics as those satisfying a certain linear growth condition on powers in a neighbourhood of 1 . This condition in turn has already been studied in the literature in the context of locally compact groups, where it turned out to be central to the solution to Hilbert's fifth problem. We shall discuss this connection after our result.

Theorem 3. The following conditions are equivalent for a compatible leftinvariant metric $d$ on a topological group $G$.

1. $d$ is minimal,

2. there is an open set $U \ni 1$ so that, for all $g \in G$ and $n \geqslant 1$,

$$
g, g^{2}, g^{3}, \ldots, g^{n} \in U \Longrightarrow d(g, 1) \leqslant \frac{1}{n},
$$

3. there is an open set $U \ni 1$ and a constant $K \geqslant 1$ so that, for all $g \in G$ and $n \geqslant 1$,

$$
g, g^{2}, g^{3}, \ldots, g^{n} \in U \Longrightarrow n \cdot d(g, 1) \leqslant K \cdot d\left(g^{n}, 1\right),
$$

4. there are constants $\varepsilon>0$ and $K \geqslant 1$ so that, for all $g \in G$ and $n \geqslant 1$,

$$
d(g, 1) \leqslant \frac{\varepsilon}{n} \Longrightarrow n \cdot d(g, 1) \leqslant K \cdot d\left(g^{n}, 1\right),
$$

5. there are an open set $U \ni 1$ and a constant $K \geqslant 1$ so that, for all $g \in G$ and $n \geqslant 0$,

$$
g, g^{2}, g^{4}, g^{8}, \ldots, g^{2^{n}} \in U \Longrightarrow 2^{n} \cdot d(g, 1) \leqslant K \cdot d\left(g^{2^{n}}, 1\right) .
$$

The above result may be said to provide a satisfactory description of minimal metrics on the group; indeed, the criterion only involves computations with powers of single elements. On the other hand, we have no informative reformulation of which metrisable groups admit minimal metrics. One would like to know if there is such a description that does not directly involve asking for an object as complicated as a minimal metric itself. More precisely, the following problem remains open.

Problem 4. Let $G$ be a universal Polish group, e.g., $G=\operatorname{Homeo}\left([0,1]^{\mathbb{N}}\right)$. Is the collection

$$
\{H \leqslant G \mid H \text { is a closed subgroup admitting a minimal metric }\}
$$

Borel in the standard Borel space of closed subgroups of $G$ ?

In connection with this, we should point out that every Polish group $G$ with a minimal metric is Weil complete and hence that every minimal metric on $G$ is complete (see Lemma 23). On the other hand, M. Malicki [9] has shown that the class of Weil complete Polish groups itself is not Borel. 
Since a compatible left-invariant metric on a topological group $H$ need not extend to a compatible left-invariant metric on a supergroup $G$, it is far from clear from the definition of minimality that the restriction of a minimal metric on $G$ to a subgroup $H$ is also minimal on $H$. However, using instead the reformulations of Theorem 3, this becomes obvious, whence the following corollary.

Corollary 5. The class of topological groups admitting minimal metrics is closed under passing to subgroups.

\section{Proof of the main theorem}

Before commencing the proof of Theorem 3, we recall some procedures for constructing compatible left-invariant metrics on a topological group. The main result in this area is the above mentioned theorem independently due to Birkhoff and Kakutani. Of the two proofs, Birkhoff's is the simplest and relies on a memorable little trick.

Lemma 6. (G. Birkhoff [1]) Let $G$ be a topological group and $\left\{V_{3^{n}}\right\}_{n \in \mathbb{Z}} a$ neighbourhood basis at the identity consisting of symmetric open sets so that $G=$ $\bigcup_{n \in \mathbb{Z}} V_{3^{n}}$ and $\left(V_{3^{n}}\right)^{3} \subseteq V_{3^{n+1}}$. Define $\delta(g, f)=\inf \left(2^{n} \mid f^{-1} g \in V_{3^{n}}\right)$ and put

$$
d(g, f)=\inf \left(\sum_{i=0}^{k-1} \delta\left(h_{i}, h_{i+1}\right) \mid h_{0}=g, h_{k}=f\right) .
$$

Then $\delta(g, f) \leqslant 2 \cdot d(g, f) \leqslant 2 \cdot \delta(g, f)$ and $d$ is a compatible left-invariant metric on $G$.

However, the metric $d$ produced by Birkhoff's construction decreases exponentially faster than needed for our purposes due to a factor $\left(\frac{3}{2}\right)^{n}$. For this, we shall instead rely on the construction of Kakutani from which a better estimate can be extracted (see [3] for a proof of the exact statement of Lemma 7 below).

Lemma 7. (S. Kakutani [7]) Let $G$ be a topological group and $\left\{V_{2^{-n}}\right\}_{n \in \mathbb{N}}$ a neighbourhood basis at the identity consisting of symmetric open sets satisfying $\left(V_{2^{-n}}\right)^{2} \subseteq V_{2^{-n+1}}$. Then there is a compatible left-invariant metric d on $G$ so that

$$
B_{d}\left(2^{-n}\right) \subseteq V_{2^{-n}} \subseteq B_{d}\left(8 \cdot 2^{-n}\right)
$$

for all $n \in \mathbb{N}$.

We now turn to the proof of Theorem 3. 
Proof. $(1) \Rightarrow(2)$ : We claim first that there is an open neighbourhood $U \ni 1$ so that, for all $k \geqslant 1$ and $g \in G$,

$$
g, g^{2}, g^{3}, \ldots, g^{2^{k}} \in U \Longrightarrow g \in B_{d}\left(2^{-k}\right) .
$$

In order to see this, we assume the contrary. Let $V_{2^{-0}}=G$ and, for $m \geqslant 1$, inductively define symmetric open sets $V_{2^{-m}} \ni 1$ as follows.

Assume that $V_{2^{-m}}$ is the last term that has been defined thus far and let $n \geqslant m$ be large enough so that $B_{d}\left(\frac{1}{n}\right) \subseteq V_{2^{-m}}$. Since the claim fails for $U=B_{d}\left(\frac{1}{n}\right)$, there are $k \geqslant 1$ and $g \notin B_{d}\left(2^{-k}\right)$ so that

$$
g, g^{2}, g^{3}, \ldots, g^{2^{k}} \in B_{d}(1 / n)
$$

Let $F=\left\{1, g, g^{-1}\right\}$ and note that, since $B_{d}\left(\frac{1}{n}\right)$ is symmetric, we have $F^{2^{k}} \subseteq B_{d}\left(\frac{1}{n}\right)$. Therefore, as $F$ is finite and $B_{d}\left(\frac{1}{n}\right)$ open, we can pick a sufficiently small symmetric open $W \ni 1$ so that also $(W F W)^{2^{k}} \subseteq B_{d}\left(\frac{1}{n}\right) \subseteq V_{2^{-m}}$. We then set

$$
V_{2^{-m-k}}=W F W, \quad V_{2^{-m-k+1}}=(W F W)^{2}, \quad \ldots \quad, V_{2^{-m-1}}=(W F W)^{2^{k-1}} .
$$

At the next stage, we begin with the term $V_{2^{-m-k}}$ and proceed as above.

Therefore, at the end of the construction, we have a sequence $G=V_{2^{-0}} \supseteq V_{2^{-1}} \supseteq$ ... of symmetric open sets forming a neighbourhood basis at 1 so that $\left(V_{2^{-m}}\right)^{2} \subseteq$ $V_{2^{-m+1}}$ for all $m \geqslant 0$. We now apply Lemma 7 to the sequence $\left(V_{2^{-m}}\right)_{m \geqslant 0}$ to obtain a compatible left-invariant metric $\partial$ satisfying

$$
B_{\partial}\left(2^{-m}\right) \subseteq V_{2^{-m}} \subseteq B_{\partial}\left(8 \cdot 2^{-m}\right) .
$$

Note now that there are infinitely many $m$ so that some stage in the construction began with the term $V_{2^{-m}}$. So fix such an $m$ and let $k, g, F$ and $W$ be as in the construction step. Then $g \in W F W=V_{2^{-m-k}} \subseteq B_{\partial}\left(8 \cdot 2^{-m-k}\right)$ and $g \notin B_{d}\left(2^{-k}\right)$, whence

$$
2^{m-3} \cdot \partial(g, 1)<2^{-k} \leqslant d(g, 1) .
$$

Therefore, $\partial$ is a compatible left-invariant metric on $G$, but id: $(G, \partial) \rightarrow(G, d)$ is not Lipschitz for short distances, contradicting the minimality of $d$ and thus proving the claim.

So, using the claim, fix $U \ni 1$ open so that, for all $g \in G$ and $k \geqslant 1$,

$$
g, g^{2}, g^{3}, \ldots, g^{2^{k}} \in U \Longrightarrow g \in B_{d}\left(2^{-k}\right)
$$

and pick some open $V \ni 1$ so that $V^{2} \subseteq U$. Now suppose $g, g^{2}, g^{3}, \ldots, g^{m} \in V$ for some $m$ and let $k \geqslant 0$ be so that $2^{k} \leqslant m<2^{k+1}$. Then also $g^{2^{k}+n}=g^{2^{k}} g^{n} \in V^{2} \subseteq U$ for all 
$n \leqslant 2^{k}$ and so $g^{i} \in U$ for all $i \leqslant 2^{k+1}$. In particular, $g \in B_{d}\left(2^{-k-1}\right) \subseteq B_{d}\left(\frac{1}{m}\right)$. In other words,

which proves (2).

$$
g, g^{2}, g^{3}, \ldots, g^{m} \in V \Longrightarrow d(g, 1) \leqslant \frac{1}{m}
$$

$(2) \Rightarrow(3)$ : Assume that $U \ni 1$ is as in (2). By shrinking $U$ if needed, we may assume that $U=B_{d}\left(\frac{1}{p}\right)$ for some integer $p \geqslant 1$. Now, choose a symmetric open $W \ni 1$ so that $W^{2 p} \subseteq U$. We claim that

$$
g, g^{2}, g^{3}, \ldots, g^{n} \in W \Longrightarrow n \cdot d(g, 1) \leqslant 4 p \cdot d\left(g^{n}, 1\right),
$$

which thus verifies (3).

Indeed, suppose $g, g^{2}, g^{3}, \ldots, g^{n} \in W$ and $g \neq 1$. Then also $g, g^{2}, g^{3}, \ldots, g^{2 p n} \in$ $W^{2 p} \subseteq U$ and thus, if $m$ is minimal so that $g^{m+1} \notin U$, we have $m \geqslant 2 p n$ and $d(g, 1) \leqslant \frac{1}{m}$. Let now $k \geqslant 1$ be such that $k n \leqslant m<m+1 \leqslant(k+1) n$. Then

$$
\begin{aligned}
d\left(g^{(k+1) n}, 1\right) & \geqslant d\left(g^{m+1}, 1\right)-d\left(g^{(k+1) n}, g^{m+1}\right) \\
& =d\left(g^{m+1}, 1\right)-d\left(g^{(k+1) n-(m+1)}, 1\right) \\
& \geqslant \frac{1}{p}-[(k+1) n-(m+1)] \cdot d(g, 1) \\
& \geqslant \frac{1}{p}-\frac{n}{m},
\end{aligned}
$$

whereby

$$
d\left(g^{n}, 1\right) \geqslant \frac{d\left(g^{(k+1) n}, 1\right)}{k+1} \geqslant \frac{\frac{1}{p}-\frac{n}{m}}{k+1} \geqslant \frac{\frac{1}{p}-\frac{n}{2 p n}}{2 k}=\frac{1}{4 p} \cdot \frac{1}{k} \geqslant \frac{1}{4 p} \cdot \frac{n}{m} \geqslant n \frac{1}{4 p} \cdot d(g, 1)
$$

as claimed.

$(3) \Rightarrow(4)$ : Let $U$ and $K$ be as in (3) and simply choose $\varepsilon>0$ so that $B_{d}(2 \varepsilon) \subseteq U$. Then $d(g, 1) \leqslant \frac{\varepsilon}{n}$ implies that $g, g^{2}, g^{3}, \ldots, g^{n} \in B_{d}(2 \varepsilon) \subseteq U$ and hence that

$$
n \cdot d(g, 1) \leqslant K \cdot d\left(g^{n}, 1\right) .
$$

$(4) \Rightarrow(3)$ : Let $\varepsilon>0$ and $K \geqslant 1$ be as in (4) and set $U=B_{d}\left(\frac{\varepsilon}{2 K}\right)$. Now, suppose $g \neq 1$ and that $g, g^{2}, \ldots, g^{n} \in U$ for some $n \geqslant 1$. Let $m \geqslant 1$ be so that $g \in B_{d}\left(\frac{\varepsilon}{m}\right) \backslash$ $B_{d}\left(\frac{\varepsilon}{m+1}\right)$. Then, by (4), we have

$$
K \cdot d\left(g^{m}, 1\right) \geqslant m \cdot d(g, 1) \geqslant \varepsilon \frac{m}{m+1} \geqslant \frac{\varepsilon}{2},
$$

i.e., $g^{m} \notin U$, whereby $m>n$. It thus follows that $d(g, 1) \leqslant \frac{\varepsilon}{n}$ and hence, by (4) again, that $n \cdot d(g, 1) \leqslant K \cdot d\left(g^{n}, 1\right)$. In other words,

$$
g, g^{2}, \ldots, g^{n} \in U \Longrightarrow n \cdot d(g, 1) \leqslant K \cdot d\left(g^{n}, 1\right) .
$$


$(3) \Rightarrow(5)$ : Fix $U$ and $K$ as in (3). By shrinking $U$ and increasing $K$, we may suppose that $U=B_{d}\left(2^{-k}\right)$ and $K=2^{k}$ for some $k \geqslant 3$. Set also $V=B_{d}\left(2^{-2 k}\right)$, whereby $V^{2^{k}} \subseteq U$. We claim that, for all $m$ and $g$,

$$
\left(\forall i \leqslant 2^{m}: g^{i} \in V\right) \&\left(\forall i \leqslant m+2 k+1: g^{2^{i}} \in V\right) \Longrightarrow\left(\forall i \leqslant 2^{m+1}: g^{i} \in V\right) .
$$

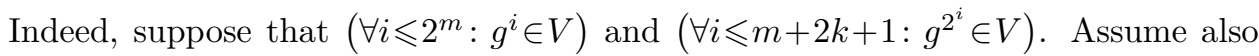
that $2^{m}<i<2^{m+2 k+1}$ is given. Write $i=2^{p_{r}}+2^{p_{r-1}}+\ldots+2^{p_{1}}+j$ for some $m \leqslant p_{1}<$ $p_{2}<\ldots<p_{r}<m+2 k+1$ and $j<2^{m}$ and note that, since $r \leqslant 2 k+1 \leqslant 2^{k}-1$, we have

$$
g^{i}=g^{2^{p_{r}}} \ldots g^{2^{p_{1}}} \cdot g^{j} \in V^{r} V \subseteq V^{2^{k}} \subseteq U .
$$

Therefore, as also $g^{2^{m+2 k+1}} \in V$, we see that $g^{i} \in U$ for all $i \leqslant 2^{m+2 k+1}$. By the hypothesis on $U$, it follows that

$$
2^{m+2 k+1} \cdot d(g, 1) \leqslant 2^{k} \cdot d\left(g^{2^{m+2 k+1}}, 1\right) \leqslant 2^{k} \cdot 2^{-k}=1,
$$

i.e., $d(g, 1) \leqslant 2^{-(m+2 k+1)}$. So, using that $d\left(g^{i}, 1\right) \leqslant i \cdot d(g, 1)$, this shows that $g^{i} \in V$ for all $i \leqslant 2^{m+1}$, proving the claim.

Put now $W=B_{d}\left(2^{-4 k}\right)$ and assume that $n$ and $g$ are given so that

$$
g, g^{2}, g^{4}, g^{8}, \ldots, g^{2^{n}} \in W .
$$

Then, since $W^{2^{2 k}} \subseteq V$, we have that $g^{2^{i}} \in V$ for all $i \leqslant n+2 k$. Using our claim to induct on $m=1,2, \ldots, n-1$, we see that $g^{i} \in V \subseteq U$ for all $i \leqslant 2^{n}$, and thus $2^{n} \cdot d(g, 1) \leqslant$ $2^{k} \cdot d\left(g^{2^{n}}, 1\right)$, which proves $(5)$.

$(5) \Rightarrow(1)$ : Suppose that $\partial$ is another compatible left-invariant metric on $G$. Fix $\varepsilon, \eta>0$ and $K \geqslant 1$ so that

$$
g, g^{2}, g^{4}, g^{8}, \ldots, g^{2^{n}} \in B_{d}(\varepsilon) \Longrightarrow 2^{n} \cdot d(g, 1) \leqslant K \cdot d\left(g^{2^{n}}, 1\right)
$$

and $B_{\partial}(\eta) \subseteq B_{d}(\varepsilon)$. Note then that, if $n \geqslant 0$ and $g \in B_{\partial}\left(\frac{\eta}{2^{n}}\right)$, we also have $g, g^{2}, g^{4}, g^{8}$, $\ldots, g^{2^{n}} \in B_{\partial}(\eta) \subseteq B_{d}(\varepsilon)$, whence

$$
2^{n} \cdot d(g, 1) \leqslant K \cdot d\left(g^{2^{n}}, 1\right) \leqslant K \varepsilon
$$

i.e., $g \in B_{d}\left(\frac{K \varepsilon}{2^{n}}\right)$. In other words, $B_{\partial}\left(\frac{\eta}{2^{n}}\right) \subseteq B_{d}\left(\frac{K \varepsilon}{2^{n}}\right)$ for all $n \geqslant 0$.

Now, if $g \in B_{\partial}(\eta)$ is any non-identity element, pick $n \geqslant 0$ so that $g \in B_{\partial}\left(\frac{\eta}{2^{n}}\right) \backslash$ $B_{\partial}\left(\frac{\eta}{2^{n+1}}\right) \subseteq B_{d}\left(\frac{K \varepsilon}{2^{n}}\right)$. Then,

$$
d(g, 1) \leqslant \frac{K \varepsilon}{2^{n}}=\frac{2 K \varepsilon}{\eta} \cdot \frac{\eta}{2^{n+1}} \leqslant \frac{2 K \varepsilon}{\eta} \cdot \partial(g, 1),
$$

showing that the map id: $\left(B_{\partial}(\eta), \partial\right) \rightarrow\left(B_{\partial}(\eta), d\right)$ is $\frac{2 K \varepsilon}{\eta}$-Lipschitz. 
The most obvious example of a minimal metric would seem to be the norm metric on the additive group $(X,+)$ of a Banach space $(X,\|\cdot\|)$. Beyond those, we look at groups of operators.

Example 8. (The unitary group) Using the spectral theorem, it is not hard to verify that the metric induced by the operator norm is minimal on the unitary group $U(\mathcal{H})$ of the separable infinite-dimensional Hilbert space $\mathcal{H}$.

Indeed, set

$$
U=\{u \in U(\mathcal{H}) \mid\|u-\mathrm{Id}\|<1\}
$$

and suppose $u, u^{2}, u^{3}, \ldots, u^{n} \in U$ for some fixed $u \in U(\mathcal{H})$. Then, by the spectral theorem, there is a $\sigma$-finite measure space $(X, \mu)$ and a measurable function $\phi: X \rightarrow \mathbb{T}$ so that $u$ is unitarily equivalent to the multiplication operator $M_{\phi}: L^{2}(X, \mu) \rightarrow$ $L^{2}(X, \mu)$ defined by

$$
\left(M_{\phi} \xi\right)(x)=\phi(x) \xi(x)
$$

As, for a multiplication operator $M_{\psi}$, we have $\left\|M_{\psi}\right\|=\|\psi\|_{\infty}$ it follows that, for $k=1, \ldots, n$,

$$
\begin{aligned}
\operatorname{ess} \sup \left\{\left|\phi(x)^{k}-1\right| \mid x \in X\right\} & =\left\|\phi^{k}-1\right\|_{\infty} \\
& =\left\|M_{\phi^{k}-1}\right\| \\
& =\left\|M_{\phi}^{k}-\mathrm{Id}\right\| \\
& =\left\|u^{k}-\mathrm{Id}\right\| \\
& <1 .
\end{aligned}
$$

Consider now a value $e^{i \alpha}=\phi(x), \alpha \in[-\pi, \pi]$, of $\phi$ so that

$$
\left|e^{i k \alpha}-1\right|=\left|\phi(x)^{k}-1\right|<1
$$

for all $k=1, \ldots, n$. Then clearly $k \alpha \in\left[-\frac{\pi}{2}, \frac{\pi}{2}\right]$ for $k=1, \ldots, n$, whence $|\alpha| \leqslant \frac{\pi}{2 n}$ and so $|\phi(x)-1|=\left|e^{i \alpha}-1\right|<\frac{4}{n}$. From this it thus follows that $|\phi(x)-1|<\frac{4}{n}$ for almost all $x \in X$ and hence $\|u-\operatorname{Id}\|<\frac{4}{n}$. So

$$
u, u^{2}, \ldots, u^{n} \in U \Longrightarrow\|u-\operatorname{Id}\|<\frac{4}{n},
$$

showing that the metric is minimal.

Example 9. (Fredholm unitary group) While $U(\mathcal{H})$ is not separable in the operator norm topology, every separable closed subgroup $G \leqslant U(\mathcal{H})$ is Polish and, by Corollary 5 , the operator norm metric will be minimal on $G$. For example, one may consider the Fredholm unitary group

$$
\{\lambda \operatorname{Id}+K \in U(\mathcal{H}) \mid \lambda \in \mathbb{T} \& K \text { a compact operator }\},
$$

which is Polish by the norm separability of the ideal of compact operators. 
Condition (4) of Theorem 3 has been studied earlier in the literature as part of the solution to Hilbert's fifth problem due to A. Gleason, D. Montgomery, H. Yamabe and L. Zippin. Indeed, in the book [15] by T. Tao, metrics satisfying this condition are termed weak Gleason as they underlie Gleason's results in [5]. In particular, in [15] it is shown that a locally compact metrisable group is a Lie group if and only if it has a weak Gleason metric. Moreover, in the locally compact setting, every weak Gleason metric is actually Gleason, meaning that it satisfies a further estimate on commutators (cf. Theorem 1.5.5 [15]). We do not know if this holds in the broader category of Polish groups.

Observation 10. (Multiplication is locally Lipschitz) Let us also mention that, if $d$ is a minimal metric on $G$, then there is a constant $K$ and an open set $V \ni 1$ so that

$$
f, g, h, k \in V \Longrightarrow d(f h, g k) \leqslant K \cdot(d(f, g)+d(h, k)),
$$

i.e., multiplication is $K$-Lipschitz in an identity neighbourhood.

To see this, fix $0<\varepsilon<1$ so that $d(g, 1) \leqslant 1 / n$ whenever $g, g^{2}, \ldots, g^{n} \in B_{d}(\varepsilon)$. Then, given distinct $g, f \in B_{d}\left(\frac{\varepsilon}{4}\right)$, let $n \geqslant 1$ be so that $g^{-1} f \in B_{d}\left(\frac{\varepsilon}{2 n}\right) \backslash B_{d}\left(\frac{\varepsilon}{2(n+1)}\right)$. So, if $h \in B_{d}\left(\frac{\varepsilon}{4}\right)$, we have $\left(h^{-1} g^{-1} f h\right)^{i}=h^{-1}\left(g^{-1} f\right)^{i} h \in B_{d}(\varepsilon)$ for $i=1, \ldots, n$, whereby

$$
d(f h, g h)=d\left(h^{-1} g^{-1} f h, 1\right) \leqslant \frac{1}{n} \leqslant \frac{2}{n+1} \leqslant \frac{4}{\varepsilon} \cdot d(f, g) .
$$

Thus, if $k \in G$ is any other element, we have

$$
d(f h, g k) \leqslant d(f h, g h)+d(g h, g k) \leqslant \frac{4}{\varepsilon} \cdot d(f, g)+d(h, k) \leqslant \frac{4}{\varepsilon} \cdot(d(f, g)+d(h, k)) .
$$

So setting $K=\frac{4}{\varepsilon}$ and $V=B_{d}\left(\frac{\varepsilon}{4}\right)$, the claim follows.

By Condition (2) of Theorem 3, it is easy to see that, if $G$ is a group with a weak Gleason metric, then $G$ is $N S S$, i.e., has no small subgroups, which simply means that there is a neighbourhood $U \ni 1$ not containing any non-trivial subgroup. Moreover, in the locally compact metrisable case, being NSS is equivalent to being a Lie group and thus also to having a weak Gleason metric (see the exposition in [10] or [15]). By Theorem 3, weak Gleason and minimal metrics coincide, but we shall prefer the latter more descriptive terminology.

Recall that two topological groups $G$ and $H$ are locally isomorphic if there are symmetric open identity neighbourhoods $U \subseteq G, V \subseteq H$ and a homeomorphism $\phi: U \rightarrow V$ so that, for all $g, f \in U$ with $g f^{-1} \in U$, we have

$$
\phi\left(g f^{-1}\right)=\phi(g) \phi(f)^{-1}
$$


and so, in particular, $\phi(1)=1$ and $\phi\left(f^{-1}\right)=\phi(f)^{-1}$. For example, if $\Gamma$ is a discrete normal subgroup of a topological group $G$, then the quotient map $\pi: G \rightarrow G / \Gamma$ is a local isomorphism between sufficiently small identity neighbourhoods.

While evidently minimal metrics are characterised locally, the existence of minimal metrics appears a priori to be a global issue.

Proposition 11. The class of topological groups admitting minimal metrics is closed under local isomorphism.

Proof. Let $\phi: U \rightarrow V$ be a local isomorphism between open identity neighbourhoods in topological groups $G$ and $H$ respectively and assume $d$ is a minimal metric on $G$. We must show that also $H$ admits a minimal metric. By rescaling $d$, we may assume that $B_{d}(1) \subseteq U$. For $n \geqslant 1$, set

$$
V_{2^{-n}}=\phi\left[B_{d}\left(2^{-n}\right)\right]
$$

and observe, since $\phi$ is a local isomorphism, that the $V_{2^{-n}}$ are symmetric open identity neighbourhoods in $H$ verifying $\left(V_{2^{-n-1}}\right)^{2} \subseteq V_{2^{-n}}$ for all $n \geqslant 1$. By Lemma 7 , we find a compatible left-invariant metric $\partial$ on $H$ so that

$$
B_{\partial}\left(2^{-n}\right) \subseteq V_{2^{-n}} \subseteq B_{\partial}\left(8 \cdot 2^{-n}\right)
$$

for all $n \geqslant 1$. Using Theorem 3 (5) and the minimality of $d$, we pick a symmetric open identity neighbourhood $W \subseteq B_{d}\left(\frac{1}{2}\right)$ and a constant $K$ so that, for all $g \in G$ and $n \geqslant 1$,

$$
g, g^{2}, g^{4}, g^{8}, \ldots, g^{2^{n}} \in W \Longrightarrow 2^{n} \cdot d(g, 1) \leqslant K \cdot d\left(g^{2^{n}}, 1\right) .
$$

Assume now that $x \in H$ satisfies $x, x^{2}, x^{4}, x^{8}, \ldots, x^{2^{n}} \in \phi[W]$ and write $x=\phi(g)$ for some $g \in W$. Then $g, g^{2} \in U$ and so $\phi\left(g^{2}\right)=\phi(g)^{2}=x^{2} \in \phi[W]$. As $\phi: U \rightarrow V$ is a bijection, we conclude that also $g^{2} \in W$. It follows again that $g^{2}, g^{4} \in U$ and so $\phi\left(g^{4}\right)=\phi\left(g^{2}\right)^{2}=x^{4} \in \phi[W]$, whence $g^{4} \in W$, et cetera. We therefore end up with

$$
g, g^{2}, g^{4}, g^{8}, \ldots, g^{2^{n}} \in W
$$

and $\phi\left(g^{2^{m}}\right)=x^{2^{m}}$ for $m \leqslant n$. In particular, $2^{n} \cdot d(g, 1) \leqslant K \cdot d\left(g^{2^{n}}, 1\right) \leqslant \frac{K}{2}$. So, if $k$ is minimal so that $K \leqslant 2^{k}$, this shows that $g \in B_{d}\left(2^{k-n}\right)$ and thus $x=\phi(g) \in$ $\phi\left[B_{d}\left(2^{k-n}\right)\right]=V_{2^{k-n}} \subseteq B_{\partial}\left(8 \cdot 2^{k-n}\right)$ provided $n>k$. In other words, for all $n>k$ and $x \in H$, we have

$$
x, x^{2}, x^{4}, x^{8}, \ldots, x^{2^{n}} \in \phi[W] \Longrightarrow \partial(x, 1)<8 \cdot 2^{k-n} .
$$

Let $O$ be a symmetric open identity neighbourhood in $H$ so that $O^{2^{k}} \subseteq \phi[W]$ and suppose that $x \in H \backslash\{1\}$ satisfies $x, x^{2}, x^{4}, x^{8}, \ldots, x^{2^{n}} \in O$ for some $n \geqslant 1$. Then, if $m$ is minimal so that $x^{2^{m+1}} \notin \phi[W]$, we must have $m>k$, whence $\partial(x, 1)<8 \cdot 2^{k-m}$. On 
the other hand, if $p \geqslant 1$ is chosen so that $B_{\partial}\left(2^{-p}\right) \subseteq \phi[W]$, we have $\partial\left(x^{2^{m+1}}, 1\right) \geqslant 2^{-p}$, i.e.,

$$
\partial(x, 1)<2^{3+k+p-m} \cdot \partial\left(x^{2^{m+1}}, 1\right) \leqslant 2^{4+k+p-n} \cdot \partial\left(x^{2^{n}}, 1\right) .
$$

In other words, for all $x \in H$ and $n \geqslant 1$,

$$
x, x^{2}, x^{4}, x^{8}, \ldots, x^{2^{n}} \in O \Longrightarrow 2^{n} \cdot \partial(x, 1) \leqslant 2^{4+k+p} \cdot \partial\left(x^{2^{n}}, 1\right),
$$

which shows that $\partial$ is a minimal metric on $H$.

\section{Maximal metrics and global Lipschitz structure}

Whereas a minimal metric establishes a canonical local Lipschitz geometry on a topological group, we will now combine this with the analysis from [13] of the corresponding problem at the large scale.

Definition 12. A compatible left-invariant metric $d$ on a topological group $G$ is said to be maximal if, for every other compatible left-invariant metric $\partial$ on $G$, the map

$$
\text { id }:(G, d) \longrightarrow(G, \partial)
$$

is Lipschitz for large distances, that is, $\partial \leqslant K \cdot d+C$ for some constants $K, C$.

Clearly, any two maximal metrics $d$ and $\partial$ on a topological group $G$ are quasiisometric, that is $\frac{1}{K} \cdot d-C \leqslant \partial \leqslant K \cdot d+C$ for some constants $K, C$.

Lemma 13. Suppose that $d$ and $\partial$ are both simultaneously minimal and maximal metrics on a topological group $G$. Then $d$ and $\partial$ are bi-Lipschitz equivalent, i.e.,

$$
\frac{1}{L} \cdot d \leqslant \partial \leqslant L \cdot d
$$

for some constant $L$.

Proof. Since $d$ is minimal, there is an identity neighbourhood $V$ and a constant $K$ so that

$$
\text { id }:(V, \partial) \longrightarrow(V, d)
$$

is $K$-Lipschitz. On the other hand, as $\partial$ is maximal, there are constants $M, N$ so that $d \leqslant M \cdot \partial+N$. It thus follows that, for $x \in G$ and $v \in V$,

$$
d(x v, x)=d(v, 1) \leqslant K \cdot \partial(v, 1)=K \cdot \partial(x v, x),
$$


while, for $a \notin V$,

$$
\begin{aligned}
d(x a, x) & \leqslant M \cdot \partial(x a, x)+N \\
& \leqslant M \cdot \partial(x a, x)+\frac{N \cdot \partial(a, 1)}{\inf (\partial(y, 1) \mid y \notin V)} \\
& \leqslant\left(M+\frac{N}{\inf (\partial(y, 1) \mid y \notin V)}\right) \cdot \partial(x a, x) .
\end{aligned}
$$

So $d \leqslant L \cdot \partial$, where $L=\max \left\{K, M+\frac{N}{\inf (\partial(y, 1) \mid y \notin V)}\right\}$ and by symmetry we find that $d$ and $\partial$ are bi-Lipschitz equivalent.

Thus, if $G$ admits a metric that is simultaneously minimal and maximal, then this defines a canonical global Lipschitz geometric structure on $G$. To characterise this situation, we need a few new concepts and results that can all be found in [13].

A topological group is Baire if it satisfies the Baire category theorem, that is, if the intersection of countably many dense open sets is dense in $G$. Also, we say that $G$ is European if it is Baire and, for every identity neighbourhood $V$, there is a countable set $D \subseteq G$ so that $G=\langle V \cup D\rangle$. Clearly every Polish group and every connected completely metrisable group, e.g., the additive group $(X,+)$ of a Banach space, is European. Also, a locally compact Hausdorff group is European if and only if it is $\sigma$-compact. For the latter fact, note that, if $G$ is $\sigma$-compact, it can be covered by countably many translates of any identity neighbourhood $V$ and vice versa, if $G$ is European and $V$ is a compact identity neighbourhood, then $G$ is covered by the compact sets $\left(F^{ \pm} V\right)^{n}$ where $F$ ranges over finite subsets of a countable set $D$ as above.

Finally, a subset $B$ of a topological group $G$ is coarsely bounded if it has finite diameter in every continuous left-invariant pseudometric on $G$. If $G$ is European, this is equivalent to asking that, for every identity neighbourhood $V$, there is a finite set $F \subseteq G$ and a $k$ so that $B \subseteq(F V)^{k}$. Also, the group $G$ is coarsely bounded if it is coarsely bounded as a subset of itself.

Now, as opposed to minimal metrics, we do have a characterisation of the existence of maximal metrics. Namely, as shown in [13], a metrisable European group $G$ admits a maximal metric $d$ if and only if it is algebraically generated by a coarsely bounded set, which furthermore may be taken to be an identity neighbourhood $V$. Moreover, in this case, the maximal metric $d$ will be quasiisometric to the word metric

$$
\rho_{V}(g, f)=\min \left(k \mid \exists v_{1}, \ldots, v_{k} \in V^{ \pm}: g=f v_{1} \ldots v_{k}\right)
$$

associated with $V$. 
Theorem 14. Let $G$ be a Polish or, more generally, a European topological group generated by a coarsely bounded set and assume that $G$ has a minimal metric. Then $G$ has a metric that is simultaneously maximal and minimal and hence $G$ has a well-defined global Lipschitz geometric structure.

Proof. Fix a minimal metric $d$ on $G$ and a coarsely bounded set $V$ generating $G$. As noted above, we may assume that $V$ is an open identity neighbourhood. Now, as shown in [13], the formula

$$
\partial(g, f)=\inf \left(\sum_{i=1}^{n} d\left(v_{i}, 1\right) \mid v_{i} \in V \& g=f v_{1} \ldots v_{n}\right)
$$

defines a compatible left-invariant metric on $G$, which is quasi-isometric to the word metric $\rho_{V}$. It thus follows that $\partial$ is quasi-isometric to a maximal metric on $G$ and therefore maximal itself.

Observe now that, if $W$ is a symmetric identity neighbourhood so that $W^{2} \subseteq V$, then any two elements of $W$ differ on the right by an element of $V$ and so the two metrics $d$ and $\partial$ agree on $W$. It therefore follows that $\partial$ is also minimal.

\section{Uniformly NSS groups}

Outside of the class of locally compact groups, the problem of determining which groups admit a minimal metric is unsolved. However, as is evident from Condition (2) of Theorem 3, a group $G$ with a minimal metric must be uniformly $N S S$ in the sense of the following definition.

Definition 15. (Uniformly NSS) A topological group $G$ is uniformly NSS if there is an open set $U \ni 1$ so that, for every open $V \ni 1$, there is some $n$ for which

$$
g, g^{2}, \ldots, g^{n} \in U \Longrightarrow g \in V .
$$

Observation 16. (Metrisability of uniformly NSS groups) As noted by P. Enflo [4], a uniformly NSS group is metrisable. Indeed, let $U \ni 1$ be as in the definition of the uniform NSS property and pick open neighbourhoods $W_{n} \ni 1$ so that $\left(W_{n}\right)^{n} \subseteq U$. Now, suppose $V \ni 1$ is open and let $n$ be such that $g \in V$ whenever $g, g^{2}, \ldots, g^{n} \in U$. Then clearly $g \in W_{n}$ implies that $g \in V$, i.e., $W_{n} \subseteq V$. Thus, the sets $W_{n}$ form a countable neighbourhood basis at 1 and $G$ is metrisable by the result of Birkhoff and Kakutani.

For other interesting facts about uniformly NSS groups, including that every Banach-Lie group is uniformly NSS, one may consult the paper [11] by S. A. Morris and V. Pestov. In particular, the authors show that uniformly NSS groups are 
locally minimal, which we shall not define here. However, by essentially the same proof, we may prove the following.

Proposition 17. Suppose $G \curvearrowright X$ is a continuous isometric action of a uniformly NSS topological group $G$, as witnessed by an identity neighbourhood $U$, on a metric space $(X, d)$. Assume also that, for some $\varepsilon>0$ and $x \in X$, we have

$$
g \notin U \Longrightarrow d(g x, x)>\varepsilon .
$$

Then the orbit map

$$
g \in G \longmapsto g x \in X
$$

is a uniform embedding of $G$ into $X$.

Proof. As the orbit map is easily seen to be uniformly continuous, to see that it is a uniform embedding of $G$ into $X$, it suffices to show that, for every identity neighbourhood $V$, there is $\eta>0$ so that $d(g x, x) \geqslant \eta$ whenever $g \notin V$. So let $V$ be given and pick $n$ so that $g \in V$ whenever $g, g^{2}, \ldots, g^{n} \in U$. Then, if $g \notin V$, there is $i \leqslant n$ so that $g^{i} \notin U$ and thus also

$$
n \cdot d(g x, x)=d\left(g^{n} x, g^{n-1} x\right)+d\left(g^{n-1} x, g^{n-2} x\right)+\ldots+d(g x, x) \geqslant d\left(g^{i} x, x\right)>\varepsilon .
$$

In other words, $d(g x, x) \geqslant \frac{\varepsilon}{n}$ for all $g \notin V$.

As is well-known, every Polish group $G$ can be seen a closed subgroup of the isometry group $\operatorname{Isom}(X, d)$ of a separable complete metric space $(X, d)$ equipped with the topology of pointwise convergence on $X$. Namely, for $X$ one may simply take the completion of $G$ in some compatible left-invariant metric on which $G$ of course acts via the extension of left-multiplication. Apart from this, many Polish groups occur naturally as groups of isometries of various metric spaces and structures. For these, Proposition 17 provides important structural information regarding those that may carry minimal metrics.

Indeed, suppose $G$ is a closed subgroup of the isometry group $\operatorname{Isom}(X, d)$ of a separable complete metric space and that $G$ carries a minimal metric $d$. Pick then an identity neighbourhood $U$ witnessing that $G$ is uniformly NSS and let $x_{1}, \ldots, x_{n} \in X$ and $\varepsilon>0$ be chosen so that $g \in U$ whenever $d\left(g x_{i}, x_{i}\right) \leqslant \varepsilon$ for all $i$. Applying Proposition 17 to the diagonal action of $G$ on $X^{n}$, we find that the map

$$
g \in G \longmapsto\left(g x_{1}, \ldots, g x_{n}\right) \in X^{n}
$$

is a uniform embedding of $G$ into $X^{n}$. In particular, for $g, g_{k} \in G$, we have

$$
g_{k} \underset{k \rightarrow \infty}{\longrightarrow} g \Longleftrightarrow g_{k}\left(x_{i}\right) \underset{k \rightarrow \infty}{\longrightarrow} g\left(x_{i}\right) \text { for each } i \leqslant n
$$

and thus the topology on $G$ is determined entirely by the action on the tuple $\left(x_{1}, \ldots, x_{n}\right)$. 


\section{Locally SIN groups}

Recall that a topological group $G$ is said to be a $S I N$ group (for small invariant neighbourhoods) if there a neighbourhood basis at the identity consisting of conjugacy invariant sets. In the context of metrisable groups, these are, by a result of $\mathrm{V}$. Klee [8], simply the groups admitting a compatible bi-invariant metric.

Proposition 18. Let $G$ be a SIN group with a minimal metric. Then $G$ admits a bi-invariant minimal metric.

Proof. Suppose $d$ is a left-invariant minimal metric on $G$ as witnessed by an open set $U \ni 1$ so that

$$
g, g^{2}, \ldots, g^{n} \in U \Longrightarrow d(g, 1) \leqslant \frac{1}{n}
$$

Since $G$ is SIN, we may assume that $U$ is conjugacy invariant. Also, replacing $d$ with $\min \{d, 1\}$, we can assume that $d \leqslant 1$. Define now a metric $\partial$ by

$$
\partial(g, h)=\sup _{f \in G} d(g f, h f),
$$

and note that, as $G$ is $\mathrm{SIN}, \partial$ is a compatible bi-invariant metric on $G$. We claim that $\partial$ is minimal. Indeed, supposing that $g, g^{2}, \ldots, g^{n} \in U$, then, for every $f \in G$, we have $f^{-1} g f,\left(f^{-1} g f\right)^{2}, \ldots,\left(f^{-1} g f\right)^{n} \in U$ and thus $d(g f, f)=d\left(f^{-1} g f, 1\right) \leqslant 1 / n$, i.e., $\partial(g, 1) \leqslant 1 / n$.

Definition 19. $G$ is a locally SIN group if there is an identity neighbourhood $\mathcal{O}$ so that the sets

$$
V^{\mathcal{O}}=\left\{g f g^{-1} \mid g \in \mathcal{O} \& f \in V\right\},
$$

where $V$ varies over identity neighbourhoods, form a neighbourhood basis at the identity.

We claim that $G$ is locally SIN if and only if the inversion map $g \mapsto g^{-1}$ is leftuniformly continuous on an open symmetric set $W \ni 1$. Indeed, suppose first that inversion is left-uniformly continuous on $W$. This means that, for all open $V \ni 1$ there is an open $U \ni 1$ so that

$$
g, f \in W \& g^{-1} f \in U \Longrightarrow g f^{-1} \in V .
$$

We let $\mathcal{O} \ni 1$ be symmetric open so that $\mathcal{O}^{2} \subseteq W$. Then, for every open $V \ni 1$, pick $U \subseteq \mathcal{O}$ as above. Then, if $g \in \mathcal{O} \subseteq W$ and $h \in U$, note that also $f=g h \in \mathcal{O} U \subseteq W$ and $g^{-1} f=h \in U$, whereby $g h g^{-1}=g f^{-1} \in V$, showing that $U^{\mathcal{O}} \subseteq V$, whence $G$ is locally SIN. 
Conversely, suppose that $G$ is locally SIN as witnessed by some symmetric open $\mathcal{O} \ni 1$. Then, if $V \ni 1$ is symmetric open, find some open $U$ with $1 \in U \subseteq \mathcal{O}$ and $U^{\mathcal{O}} \subseteq V$ and note that

$$
g, f \in \mathcal{O} \& g^{-1} f \in U \Longrightarrow f g^{-1}=g \cdot g^{-1} f \cdot g^{-1} \in V \Longrightarrow g f^{-1}=\left(f g^{-1}\right)^{-1} \in V .
$$

So inversion is left-uniformly continuous on $\mathcal{O}$.

Similarly, one may show that $G$ is locally SIN if and only if there is an open set $W \ni 1$ so that the map $(g, f) \in W \times W \mapsto g f \in W^{2}$ is left-uniformly continuous.

For the next proposition, recall from Section 3 that a European group $G$ is coarsely bounded exactly when, for every open $\mathcal{O} \ni 1$, there are a finite set $F \subseteq G$ and a $k$ so that $G=(F \mathcal{O})^{k}$. Apart from compact groups, this is a surprisingly common phenomenon among non-locally compact Polish or European groups, see, e.g., [12] for a wide range of examples. Of particular interest to us is the unitary group $U(\mathcal{H})$ equipped with the operator norm topology for which coarse boundedness is an immediate consequence of the spectral theorem.

Proposition 20. Let $G$ be a coarsely bounded, locally SIN, European group. Then $G$ is SIN.

Proof. Let $\mathcal{O}$ be an open identity neighbourhood witnessing that $G$ is locally SIN and pick $k$ and a finite subset $F \subseteq G$ so that $G=(F \mathcal{O})^{k}$. Suppose now that $U \ni 1$ is open. Set $V=\bigcap_{f \in F} f^{-1} U f$, which is an open neighbourhood of 1 , and let $W \ni 1$ be an open set so that $W^{\mathcal{O}} \subseteq V$, whence $W^{F \mathcal{O}}=\left(W^{\mathcal{O}}\right)^{F} \subseteq V^{F} \subseteq U$. Thus, by induction, we can choose open $U=W_{0} \supseteq W_{1} \supseteq W_{2} \supseteq \ldots \supseteq W_{k} \ni 1$ so that $W_{i}^{F \mathcal{O}} \subseteq$ $W_{i-1}$, whereby $W_{k}^{G}=W_{k}^{(F \mathcal{O})^{k}} \subseteq W_{0}=U$. In particular, $W_{k}^{G}$ is a conjugacy invariant identity neighbourhood contained in $U$, which shows that $G$ admits a neighbourhood basis at 1 consisting of conjugacy invariant sets.

Observation 21. (Uniformly NSS groups are locally SIN) Enflo [4] showed that uniformly NSS groups are locally SIN (though he used the terminology locally uniform in place of locally $S I N)$. To see this, let $U \ni 1$ be the open set given by the uniform NSS property and pick a symmetric open $\mathcal{O} \ni 1$ so that $\mathcal{O}^{3} \subseteq U$. Suppose now $W$ is an arbitrary neighbourhood of 1 and find $n$ so that

$$
g, g^{2}, \ldots, g^{n} \in U \Longrightarrow g \in W .
$$

We now choose some open $V \ni 1$ so that $V^{n} \subseteq \mathcal{O}$, whence also $\mathcal{O} V^{n} \mathcal{O}^{-1} \subseteq U$. In particular, if $v \in V$ and $g \in \mathcal{O}$, then $\left(g v g^{-1}\right)^{m}=g v^{m} g^{-1} \in U$ for $m=1, \ldots, n$, whereby $g v g^{-1} \in W$. In other words, $V^{\mathcal{O}} \subseteq W$, verifying that $G$ is locally SIN.

So combining this result of Enflo with Proposition 18 and Proposition 20, we obtain the following. 
Corollary 22. Every coarsely bounded European group with a minimal metric has a bi-invariant minimal metric.

Recall that a sequence $\left(f_{i}\right)$ in a metrisable group $G$ is left-Cauchy if $f_{i}^{-1} f_{j} \underset{i, j \rightarrow \infty}{\longrightarrow} 1$ and right-Cauchy if $f_{i} f_{j}^{-1} \underset{i, j \rightarrow \infty}{\longrightarrow} 1$. The group $G$ is Raikov complete if every sequence that is both left and right-Cauchy is convergent. This is equivalent to $G$ being completely metrisable, i.e., that the topology on $G$ can be induced by a complete metric. Also, $G$ is Weil complete if every left-Cauchy sequence in $G$ is convergent. This, in turn, is equivalent to the existence of a compatible complete left-invariant metric on $G$ (such groups are sometimes denoted $C L I$ for complete left-invariant). Moreover, in this case, every compatible left-invariant metric is complete.

Lemma 23. If $G$ is locally SIN and completely metrisable, then $G$ is Weil complete. In particular, every minimal metric on a completely metrisable group is necessarily complete.

Proof. Indeed, suppose that $\mathcal{O} \ni 1$ is an open set witnessing that $G$ is locally SIN and that $\left(f_{i}\right)$ is left-Cauchy. To see that $\left(f_{i}\right)$ is right-Cauchy, fix a neighbourhood $U$ of 1 and pick some $i_{0}$ so that $f_{i}^{-1} f_{j} \in \mathcal{O}$ for all $i, j \geqslant i_{0}$. Now choose some open $W \ni 1$ so that $f_{i_{0}} W f_{i_{0}}^{-1} \subseteq U$ and some open $V \ni 1$ so that $V^{\mathcal{O}} \subseteq W$. Finally, let $i_{1} \geqslant i_{0}$ be so that $f_{i}^{-1} f_{j} \in V$ whenever $i, j \geqslant i_{1}$. Then $i, j \geqslant i_{1}$ implies that $f_{i_{0}}^{-1} f_{i} \in \mathcal{O}$ and so $f_{i}=f_{i_{0}} g$ for some $g \in \mathcal{O}$, whence

$$
f_{j} f_{i}^{-1}=f_{i} \cdot f_{i}^{-1} f_{j} \cdot f_{i}^{-1}=f_{i_{0}} g \cdot f_{i}^{-1} f_{j} \cdot g^{-1} f_{i_{0}}^{-1} \in f_{i_{0}} g V g^{-1} f_{i_{0}}^{-1} \subseteq f_{i_{0}} W f_{i_{0}}^{-1} \subseteq U .
$$

Thus $\left(f_{i}\right)$ is also right-Cauchy and therefore convergent in $G$.

By Corollary 22 and the fact that every group with a minimal metric is NSS, every coarsely bounded Polish group with a minimal metric must be both NSS and SIN. Since coarsely bounded groups are very common among non-locally compact Polish groups, this provides a significant restriction on potential examples.

\section{Minimal metrics and 1-parameter subgroups}

The next result has a long history and many variations. The first occurrence seems to be the paper by Gleason [6] in which it is proved that, in a locally euclidean NSS group, there is an identity neighbourhood in which square roots, whenever they exist, are necessarily unique. We shall need a stronger version of this, namely that in a uniformly NSS group the extraction of square roots, whenever they exist, is left-uniformly continuous. A result of this form, under additional hypotheses, is also proved in Enflo's paper [4]. 


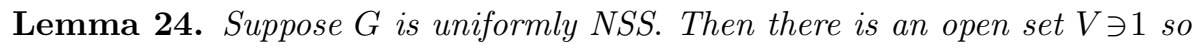
that, for every open $U \ni 1$, there is an open $W \ni 1$ so that

$$
g, f \in V \& g^{-2} f^{2} \in W \Longrightarrow g^{-1} f \in U \text {. }
$$

In particular, the map $g \mapsto g^{2}$ is injective on $V$.

Proof. Since, by Observation 21, uniformly NSS groups are also locally SIN, we fix a symmetric open set $\mathcal{O} \ni 1$ witnessing both that $G$ is uniformly NSS and locally SIN. Let also $V \ni 1$ be symmetric open so that $\left(V^{\mathcal{O}} V V\right)^{2} \subseteq \mathcal{O}$.

To see that the lemma holds for $V$, suppose $U$ is given and pick some $n$ so that $\left(y, y^{2}, \ldots, y^{n} \in \mathcal{O} \Rightarrow y \in U\right)$. Let also $W \ni 1$ be an open set with $W W^{\mathcal{O}} W^{\mathcal{O}^{2}} \ldots$ $W^{\mathcal{O}^{n-1}} \subseteq V$. Then, for $x, y \in \mathcal{O}$,

$$
x^{-i} y^{i}=\left[x^{-1} y\right] \cdot\left[y^{-1}\left(x^{-1} y\right) y\right] \cdot\left[y^{-2}\left(x^{-1} y\right) y^{2}\right] \ldots\left[y^{-(i-1)}\left(x^{-1} y\right) y^{i-1}\right]
$$

and so, if also $x^{-1} y \in W$ and $i \leqslant n$, then $x^{-i} y^{i} \in W W^{\mathcal{O}} W^{\mathcal{O}^{2}} \ldots W^{\mathcal{O}^{i-1}} \subseteq V$. In other words,

$$
x, y \in \mathcal{O} \& x^{-1} y \in W \Longrightarrow \forall i \leqslant n: x^{-i} y^{i} \in V .
$$

Suppose that $g, f \in V$ satisfy $g^{-2} f^{2} \in W$ and set $y=g^{-1} f$ and $x=g^{-1} y^{-1} g$. Then $x^{-1} y=g^{-2} f^{2} \in W$ and thus $g^{-1} y^{i} g y^{i}=x^{-i} y^{i} \in V$ for $i \leqslant n$. Note now that

$$
y^{i} \in \mathcal{O} \Longrightarrow y^{2 i}=\left(y^{i} g^{-1} y^{-i}\right) \cdot g \cdot\left(g^{-1} y^{i} g y^{i}\right) \in V^{\mathcal{O}} V V
$$

for all $i \leqslant n$. We claim that $y^{i} \in V^{\mathcal{O}} V V$ for even $i \leqslant n$ and $y^{i} \in\left(V^{\mathcal{O}} V V\right)^{2} \subseteq \mathcal{O}$ for odd $i \leqslant n$. This is clear for $i=0,1$, so suppose the result holds for all $i \leqslant j<n$ and consider $i=j+1$. If $i$ is odd, then $j$ is even and thus $y^{j} \in V^{\mathcal{O}} V V$ and $y^{i}=y y^{j} \in V^{2} \cdot V^{\mathcal{O}} V V \subseteq$ $\left(V^{\mathcal{O}} V V\right)^{2}$. On the other hand, if $i$ is even, then $l=\frac{i}{2} \leqslant j$ and so $y^{l} \in\left(V^{\mathcal{O}} V V\right)^{2} \subseteq \mathcal{O}$, whence $y^{i}=y^{2 l} \in V^{\mathcal{O}} V V$.

Thus, $y, y^{2}, \ldots, y^{n} \in \mathcal{O}$, whereby $g^{-1} f=y \in U$ as claimed.

Our final result originates in work of C. Chevalley [2], who showed how to construct one-parameter subgroups in locally Euclidean NSS groups. Again, a generalisation to the non-locally compact setting was obtained by Enflo in [4], in which he proved the result below under the assumption that the group is uniformly dissipative. However, this assumption excludes, for example, compact Lie groups and therefore does not generalise the classical setting of locally compact Lie groups. Our result below includes this latter setting.

Theorem 25. Suppose $G$ is a completely metrisable topological group admitting a minimal metric and that, for every open $W \ni 1$, the set $\left\{g^{2} \mid g \in W\right\}$ is dense in a neighbourhood of 1 . Then there are open sets $\mathcal{U} \supseteq \mathcal{O} \ni 1$ so that, for every $f \in \mathcal{O}$, there is a unique one-parameter subgroup $\left(h^{\alpha}\right)_{\alpha \in \mathbb{R}}$ with $h^{1}=f$ and $h^{\alpha} \in \mathcal{U}$ for all $\alpha \in[-1,1]$. 
Proof. Let $d$ be a minimal metric and observe that $d$ is complete by Lemma 23 . We claim that, for all identity neighbourhoods $V$, there is an identity neighbourhood $W$ so that every element of $W$ has a square root in $V$.

So, let $V$ be a given identity neighbourhood and note that, since $G$ has a minimal metric, it is uniformly NSS. Therefore, by shrinking $V$, we can by Lemma 24 suppose that, for every open $U \ni 1$, there is an open $\widetilde{U} \ni 1$ satisfying

$$
g, f \in V \& g^{-2} f^{2} \in \widetilde{U} \Longrightarrow g^{-1} f \in U .
$$

By shrinking $V$ further, we may suppose that $V$ is closed.

Let now $W=\overline{\left\{g^{2} \mid g \in V\right\}}$, which by assumption is a neighbourhood of 1 , and assume that $f \in W$. We pick $g_{n} \in V$ so that $g_{n}^{2} \underset{n}{\longrightarrow} f$, which means that $\left(g_{n}^{2}\right)$ is left-Cauchy and thus, by the above assumption (1) on $V$, also $\left(g_{n}\right)$ is left-Cauchy. Since $d$ is a complete left-invariant metric and $V$ is closed, it follows that $\left(g_{n}\right)$ is convergent to some $g \in V$, whence $g^{2}=\lim _{n} g_{n}^{2}=f$. In other words, every element of $W$ has a square root in $V$, which proves our claim.

Since $d$ is minimal, there are an identity neighbourhood $V_{0}$ and a $k \geqslant 1$ so that, for all $g \in G$ and $n \geqslant 1$,

$$
g, g^{2}, g^{4}, g^{8}, \ldots, g^{2^{n}} \in V_{0} \Longrightarrow d(g, 1) \leqslant 2^{k-n-1} \cdot d\left(g^{2^{n}}, 1\right) .
$$

Define inductively identity neighbourhoods $V_{0} \supseteq V_{1} \supseteq \ldots \supseteq V_{k}$ so that every element of $V_{i+1}$ is the square of some element in $V_{i}$. By shrinking $V_{k}$, we may assume that $V_{k}=B_{d}(\varepsilon)$ for some $\varepsilon>0$.

Now suppose that $h_{0} \in V_{k}$ and choose inductively $h_{i} \in V_{k-i}$ so that $h_{i+1}^{2}=h_{i}$. Then $h_{k}, h_{k}^{2}=h_{k-1}, h_{k}^{4}=h_{k-2}, \ldots, h_{k}^{2^{k}}=h_{0}$ all belong to $V_{0}$, whence

$$
d\left(h_{k}, 1\right) \leqslant 2^{k-k-1} \cdot d\left(h_{k}^{2^{k}}, 1\right)=1 / 2 \cdot d\left(h_{0}, 1\right) \leqslant \varepsilon / 2 .
$$

This shows that every $h \in V_{k}$ has a $2^{k}$-th root $f \in V_{k}$ so that $d(f, 1) \leqslant 1 / 2 \cdot d(h, 1)$.

Therefore, if $f \in V_{k}$ is given, we can choose an infinite sequence $h_{0}, h_{1}, h_{2}, \ldots \in V_{k}$ beginning at $h_{0}=f$ so that $h_{i+1}^{2^{k}}=h_{i}$ and $d\left(h_{i+1}, 1\right) \leqslant 1 / 2 \cdot d\left(h_{i}, 1\right)$ for all $i$. In particular, $h_{i}^{2^{k \cdot j}}=h_{i-j}$ for all $j \leqslant i$. For every dyadic rational number $\alpha=\frac{m}{2^{k \cdot i}}$ with $m \in \mathbb{Z}$ and $i \in \mathbb{N}$, we can then unambigously define $h^{\alpha}=h_{i}^{m}$ and see that $h^{\alpha} \cdot h^{\beta}=h^{\alpha+\beta}$ for all dyadic rationals $\alpha$ and $\beta$.

Now, if $\alpha \in[0,1[$ is a dyadic rational, write

$$
\alpha=\frac{a_{1}}{2^{k}}+\frac{a_{2}}{2^{k \cdot 2}}+\ldots+\frac{a_{p}}{2^{k \cdot p}}
$$

with $0 \leqslant a_{i}<2^{k}$. Then

$$
h^{\alpha}=h_{1}^{a_{1}} \cdot h_{2}^{a_{2}} \ldots h_{p}^{a_{p}}
$$


whereby

$$
\begin{aligned}
d\left(h^{\alpha}, 1\right) & \leqslant a_{1} \cdot d\left(h_{1}, 1\right)+a_{2} \cdot d\left(h_{2}, 1\right)+\ldots+a_{p} \cdot d\left(h_{p}, 1\right) \\
& \leqslant\left(\frac{a_{1}}{2}+\frac{a_{2}}{2^{2}}+\ldots+\frac{a_{p}}{2^{p}}\right) \cdot \varepsilon \\
& <2^{k} \cdot \varepsilon .
\end{aligned}
$$

Moreover, if $\alpha<\frac{1}{2^{k i}}$, then $a_{1}=\ldots=a_{i}=0$, whence $d\left(h^{\alpha}, 1\right)<2^{k-i} \cdot \varepsilon$.

It follows that the mapping $\alpha \mapsto h^{\alpha}$ is a continuous homomorphism from the additive group of dyadic rationals with the topology induced by $\mathbb{R}$ into $G$. Since $d$ is a complete metric on $G$, it follows that this extends to a continuous one-parameter subgroup $\left(h^{\alpha}\right)_{\alpha \in \mathbb{R}}$ with $h^{1}=f$ and so that $d\left(h^{\alpha}, 1\right) \leqslant 2^{k} \cdot \varepsilon$ for all $\alpha \in[-1,1]$.

Now, suppose that $\left(h^{\alpha}\right)_{\alpha \in \mathbb{R}}$ and $\left(g^{\alpha}\right)_{\alpha \in \mathbb{R}}$ are distinct one-parameter subgroups in $G$ with $h^{1}=g^{1}$. Then, by the density of the dyadic rationals in $\mathbb{R}$, there must be some dyadic rational $\alpha=\frac{1}{2^{n}}$ so that $h^{\alpha} \neq g^{\alpha}$. However, as $h^{1}=g^{1}$, it follows that there is an $\ell \geqslant 0$ so that $h^{\frac{1}{2^{\ell+1}}} \neq g^{\frac{1}{2^{\ell+1}}}$, while $h^{\frac{1}{2^{\ell}}}=g^{\frac{1}{2^{\ell}}}$. So the squaring map $f \mapsto f^{2}$ fails to be injective on any set containing $\left\{h^{\beta}\right\}_{\beta \in[-1,1]}$ and $\left\{g^{\beta}\right\}_{\beta \in[-1,1]}$.

Therefore, if we choose $V_{k}=B_{d}(\varepsilon)$ small enough so that the squaring map is injective on $B_{d}\left(2^{k} \cdot \varepsilon\right)$, which is possible by Lemma 24 , then, for every $f \in V_{k}$, there is a unique one-parameter subgroup $\left(h^{\alpha}\right)_{\alpha \in \mathbb{R}} \subseteq G$ so that $h^{1}=f$ and $h^{\alpha} \in B_{d}\left(2^{k} \cdot \varepsilon\right)$ for all $\alpha \in[-1,1]$. Setting $\mathcal{U}=B_{d}\left(2^{k} \cdot \varepsilon\right)$ and $\mathcal{O}=B_{d}(\varepsilon)$, the theorem follows.

\section{References}

1. Birkhoff, G., A note on topological groups, Compos. Math. 3 (1936), 427-430.

2. Chevalley, C., On a theorem of Gleason, Proc. Amer. Math. Soc. 2 (1951), 122-125.

3. Diestel, J. and Spalsbury, A., The Joys of Haar Measure, Am. Math. Soc., Providence, RI, 2014.

4. Enflo, P., Uniform structures and square roots in topological groups I, Israel J. Math. 8 (1970), 230-252.

5. Mattei Gleason, A., Groups without small subgroups, Ann. of Math. (2) 56 (1952), 193-212.

6. Mattei Gleason, A., Square roots in locally Euclidean groups, Bull. Amer. Math. Soc. 55 (1949), 446-449.

7. Kakutani, S., Über die Metrisation der topologischen Gruppen, in Proc. Imp. Acad. Tokyo 12 (1936), 82-84, Selected Papers, vol. 1, pp. 60-62, Birkhäuser, Basel, 1986.

8. KLEE, V. L. Jr., Invariant metrics in groups (solution of a problem of Banach), Proc. Amer. Math. Soc. 3 (1952), 484-487.

9. Maciej, M., On Polish groups admitting a compatible complete left-invariant metric, J. Symbolic Logic 76 (2011), 437-447.

10. Deane, M. and Zippin, L., Topological Transformation Groups, Reprint of the 1955 original, Krieger, Huntington, N.Y., 1974. 
11. Morris, S. A. and Pestov, V., On Lie groups in varieties of topological groups, Colloq. Math. 78 (1998), 39-47.

12. Rosendal, C., A topological version of the Bergman property, Forum Math. 21 (2009), 299-332.

13. Rosendal, C., Coarse geometry of topological groups, book manuscript 2017.

14. Sullivan, D., Hyperbolic geometry and homeomorphisms, in Geometric Topology: Proc. Topology Conf. at Athens, Ga., 1977, pp. 543-555, Academic Press, New York, 1979.

15. TAO, T., Hilbert's Fifth Problem and Related Topics, Graduate Studies in Mathematics 153, Am. Math. Soc., Providence, RI, 2014.

Christian Rosendal

Department of Mathematics, Statistics, and Computer Science (M/C 249)

University of Illinois at Chicago

851 S. Morgan St.

Chicago

IL 60607-7045

U.S.A.

and

Department of Mathematics

University of Maryland

4176 Campus Drive - William E. Kirwan

Hall

College Park

MD 20742-4015

U.S.A.

rosendal.math@gmail.com

http://homepages.math.uic.edu/ rosendal

Received November 12, 2016

in revised form July 2, 2017 